ORIGINAL ARTICLE

\title{
Childhood and adolescence poisoning in NSW, Australia: an analysis of age, sex, geographic, and poison types
}

\section{T Lam}

Injury Prevention 2003;9:338-342

Correspondence to: Dr Lawrence T Lam, Royal Alexandra Hospital for Children, Locked Bag 4001, Westmead, NSW 2145, Australia; lawrencl@chw.edu.au
Objective: This study aims to investigate whether there is any association between the types of poison substances and geographic locations for different age groups and sex.

Design: This is a population based epidemiological study utilising routinely collected inpatient statistics. Setting: Data are collected as part of the routine vital health information system via all hospitals in New South Wales (NSW), Australia.

Patients: All patients aged between 0-19 years who were admitted to a hospital because of poisoning by the four major types of substances that were defined in the study in NSW in 2000.

Main results: The standardised incidence ratios of poisoning related hospitalisation between metropolitan and rural areas varied across different poison types when compared with the NSW average. While there are few differences between metropolitan and rural areas for analgesic and chemical related poisoning admissions across different age groups and sex, differences in the standardised incidence ratios between geographic locations for psychotropic and venom related poisoning admissions were found. No significant difference in standardised mortality ratios were found between metropolitan and rural areas except for females in the 10-14 years age group (standardised mortality ratio $3.24,95 \%$ confidence interval 1.69 to 6.21 ).

Conclusions: The results obtained in this study, on the whole, provide some evidence for an association between poison types and geographic locations for psychotropic and venom related poisoning.
$P$ oisoning has been identified as one of the major causes of childhood and adolescence hospital emergency presentations and admissions in most developed countries including the United States, United Kingdom, and Australia. ${ }^{1-10}$

In developing countries, poisoning has also been recognised as a major health problem among children and adolescents. ${ }^{11-13}$ Ahmed and Andersson compared the mortality rates of unintentional injuries, including poisoning, in countries with different income levels, and found that poisoning is the second most common cause of death in all lower and upper middle income countries among the 15-24 year age group. ${ }^{12}$

Certain poison types of medicinal and non-medicinal substances have been identified as common agents in poisoning in children and adolescents. ${ }^{14-17}$ Riordan et al reviewed the literature on childhood poisoning and identified several groups of medicinal substances as the most common. ${ }^{14}$ These included analgesics, anti-inflammatory agents, psychotropic drugs such as antidepressants, and benzodiazepines related agents. Non-medicinal substances include chemicals such as organophosphates, pesticides, insecticides, organic solvents, and household cleaning products such as bleach and caustics and were also found to be common causes of poisoning among children. ${ }^{16}$ Poisoning due to psychotropic medicine was most common among adolescents. ${ }^{17}$

It has also been suggested that there is a differential relationship between poison types and geographic locations where poisoning incidences occur. ${ }^{10}$ Assumption on exposure, which is based on accessibility to poisonous substances, leads to the hypothesis that certain types of poisoning would be more prevalent in specific geographic locations. For example, children and adolescents in rural areas would have more chance of exposure to agricultural and other chemicals, hence poisoning due to these types of substances should be more prevalent in rural areas. The same argument can be applied to other drug types, especially those "over-the-counter" drugs such as paracetamol, and their associations with urban areas. Should such a hypothesis be true, there might be a need for different preventive strategies for poisoning in different geographic locations. While this is logical, it requires substantiating evidence.

The aim of this study was to investigate whether there was any association between type of poison and geographic location for different age groups and sex.

\section{METHODS}

This population based epidemiological study was conducted in New South Wales (NSW), Australia. The study utilised statewide hospitalisation data that are part of the Inpatient Statistics Collection (ISC), routinely collected by the NSW Department of Health. ${ }^{18}$ The data obtained from the ISC consisted of one full calendar year of hospital admission information in 2000. New South Wales is the largest state in Australia with an estimated population of about 6.5 million in 2000. Among these, about 1.76 million (27\%) were children and adolescents aged 0-19 years.

Subjects included in this study were children and adolescents, aged 0-19 years, admitted to hospital because of poisoning in 2000. These patients were identified from the ISC data bank if they had a primary diagnosis of poisoning according to the International Classification of Diseases, 9th revision, clinical modification (ICD-9-CM). ${ }^{19}$ Patients with diagnostic classifications ranging from 960.00 to 989.90 were included in the study.

Abbreviations: ICD-9-CM, International Classification of Diseases, 9th revision, clinical modification; ISC, Inpatient Statistics Collection; NSW, New South Wales 
Unidentified information on individual patients who were admitted to a NSW hospital because of poisoning within the study period was obtained from the NSW Department of Health. This information included age, sex, residential postcode, ICD-9-CM diagnostic classification of admitting problems, length of stay in hospital, and the outcome of hospitalisation, namely died or survived.

Poison types were classified according to the ICD-9-CM classification system, with ICD codes ranging from 960.0 to 979.9 as poisoning by drugs, medicinal and biological substances, and codes ranging from 980.0 to 989.9 as the toxic effects of non-medicinal substances. In this study, a special focus was placed on four major types of medicinal and non-medicinal substances. These four types of substances were identified in the literature as most commonly responsible for accidental poisoning among children and adolescents. They were (1) analgesics, antipyretics, and antirheumatics; (2) psychotropic drugs; (3) venom of snakes, spiders, and insects; and (4) chemicals including organophosphates, pesticides, insecticides, organic solvents, and corrosive and caustic agents.

The geographic location for each individual patient was classified according to the residential postcode. According to the NSW health areas system, the state is divided into nine greater metropolitan health areas and eight rural health regions. Each health area and region covered distinct geographical and postal regions. Hence, the residential postcode could be used as an indicator as to whether the patient belonged to a greater metropolitan health area or a rural health region. A broad classification of "metropolitan" and "rural" was used to designate the place of poisoning. Data on the estimated population size by ages and postcodes for the year 2000 were obtained from the Australian Bureau of Statistics. ${ }^{20}$

Since the aim of the study was to investigate whether there is any association between poison types and geographic locations for different age groups and sex, the focus of data analysis was on comparisons of hospitalisation rates between metropolitan and rural areas for different poison types stratified by age and sex. Standardised incidence ratios and their corresponding 95\% confidence intervals were calculated by age, sex, geographic locations, and poison types according to the method described by Rothman and Greenland. ${ }^{21}$ The standardised mortality ratios by age, sex, and geographic locations were also calculated.

\section{RESULTS}

There were 2516 children and adolescents, aged 0-19 years, admitted to hospital because of poisoning in NSW in 2000 (table 1). Of these, 1696 were poisoned by one of the four major types of substance defined above. This represented the majority (67.4\%) of total annual hospitalisations because of poisoning for the same age range in NSW. The results are summarised in table 2. Of these 1696 admissions, adolescents aged 15-19 years accounted for half of the hospitalisations $(\mathrm{n}=944,55.7 \%)$ with females having a rate of hospitalisation (392.1/100 000 population) three times that of males (130.3/ 100000 population). Among children under the age of 15 years, the 0-4 years age group was most at risk of poisoning with the highest rates of hospitalisation for both boys and girls (boys: 93.5/100 000 population, girls: 90.3/100 000 population) compared with other age groups. The total hospitalisation rate for females was higher than that of males with 120.0 and 74.1/100 000 population, respectively. Poisoning by analgesics, antipyretics, and antirheumatics type accounted for the highest frequency $(n=730)$ with 519 $(71.1 \%)$ incidences caused by paracetamol and related pain killers. This was followed by psychotropic drugs with 590 $(34.8 \%)$ admissions to hospitals. Among these admissions,
Table 1 Frequency distribution of hospitalisations due to poisoning among children and adolescents aged 0-19 years in NSW, Australia, $2000(n=2516)$

\begin{tabular}{|c|c|c|}
\hline Poison & Frequencies & $\%$ \\
\hline Analgesic/antipyretic drugs & 730 & 29.0 \\
\hline Psychotropic drugs & 590 & 23.4 \\
\hline Venoms & 293 & 11.6 \\
\hline $\begin{array}{l}\text { Organophosphates, pesticides, } \\
\text { corrosives, etc }\end{array}$ & 83 & 3.3 \\
\hline Anticonvulsant/antiparkinsonian drugs & 80 & 3.2 \\
\hline Antihypertensive drugs & 74 & 2.9 \\
\hline Primarily systemic agents & 60 & 2.4 \\
\hline Alcohol & 55 & 2.2 \\
\hline Noxious substances as food & 48 & 1.9 \\
\hline Gases, fumes, or vapours & 40 & 1.6 \\
\hline $\begin{array}{l}\text { Agents affecting smooth skeletal } \\
\text { muscles and respiratory function }\end{array}$ & 39 & 1.6 \\
\hline Agents affecting blood constituents & 33 & 1.3 \\
\hline Agents affecting mucous membrane & 31 & 1.2 \\
\hline Agents affecting gastrointestinal tract & 30 & 1.2 \\
\hline Agents affect autonomic nervous system & 26 & 1.0 \\
\hline Hormones and synthetic agents & 24 & 1.0 \\
\hline Agents affecting metabolism & 21 & 0.8 \\
\hline Antibiotics & 19 & 0.8 \\
\hline Solvents & 15 & 0.6 \\
\hline Petroleum & 14 & 0.6 \\
\hline Anti-infection agents & 8 & 0.3 \\
\hline Other metals & 6 & 0.2 \\
\hline Carbon monoxide & 6 & 0.2 \\
\hline Sedative and hypnotics & 5 & 0.2 \\
\hline Other substances unspecified & 186 & 7.4 \\
\hline Total & 2516 & 100 \\
\hline
\end{tabular}

$218(36.9 \%)$ were antidepressant related and 157 (26.6\%) were related to benzodiazepine based tranquillisers. The majority of hospitalisations occurred in metropolitan areas

Table 2 Frequency distributions of patients' characteristics and rates (per 100000 population) of hospitalisations due to poisoning with major poison types among children and adolescents aged 0-19 years in NSW, Australia $(n=1696)$

\begin{tabular}{|c|c|c|}
\hline Characteristics & Frequency & $\%$ \\
\hline \multicolumn{3}{|l|}{ Age group (years) } \\
\hline $0-4$ & 397 & 23.4 \\
\hline $5-9$ & 108 & 6.4 \\
\hline $10-14$ & 247 & 14.6 \\
\hline $15-19$ & 944 & 55.7 \\
\hline \multicolumn{3}{|l|}{ Sex } \\
\hline Male & 668 & 39.4 \\
\hline Female & 1028 & 60.6 \\
\hline \multicolumn{3}{|l|}{ Poison types } \\
\hline Analgesics & 730 & 43.0 \\
\hline Psychotropics & 590 & 34.8 \\
\hline Venoms & 293 & 17.3 \\
\hline Chemicals* & 83 & 4.9 \\
\hline \multicolumn{3}{|l|}{ Geographic location } \\
\hline Metropolitan & 1213 & 71.6 \\
\hline Rural & 481 & 28.4 \\
\hline \multicolumn{3}{|c|}{ Length of stay in hospital (days) } \\
\hline$>3$ & 116 & 6.8 \\
\hline$\leqslant 3$ & 1580 & 93.2 \\
\hline \multicolumn{3}{|l|}{ Mortality } \\
\hline Died & 98 & 5.8 \\
\hline \multirow[t]{2}{*}{ Survived } & 1598 & 94.2 \\
\hline & \multicolumn{2}{|c|}{ Rate/100 000 population } \\
\hline Age group (years) & M & $F$ \\
\hline $0-4$ & 93.5 & 90.3 \\
\hline $5-9$ & 30.1 & 17.9 \\
\hline $10-14$ & 43.3 & 70.9 \\
\hline $15-19$ & 130.3 & 302.1 \\
\hline
\end{tabular}


Table 3 Frequency distributions of major poison types by age and sex

\begin{tabular}{|c|c|c|c|c|c|c|c|c|}
\hline \multirow[b]{2}{*}{ Poison types } & \multicolumn{2}{|l|}{$0-4$ years } & \multicolumn{2}{|l|}{$5-9$ years } & \multicolumn{2}{|c|}{$10-14$ years $\dagger$} & \multicolumn{2}{|l|}{$15-19$ years $\dagger$} \\
\hline & M & $\mathbf{F}$ & M & $\mathbf{F}$ & M & $\mathbf{F}$ & $M$ & $\mathbf{F}$ \\
\hline Analgesics & $88(42.5)^{*}$ & $72(37.9)$ & $3(4.3)$ & $3(7.7)$ & 12 (12.4) & $85(56.7)$ & $122(41.4)$ & $345(53.2)$ \\
\hline Psychotropics & $57(27.5)$ & 61 (32.1) & $5(7.2)$ & 7 (17.9) & $30(30.9)$ & $36(24.0)$ & $125(42.4)$ & $269(41.4)$ \\
\hline Venoms & $32(15.5)$ & $31(16.3)$ & $58(84.1)$ & $28(71.8)$ & $55(56.7)$ & $27(18.0)$ & $42(14.2)$ & $20 \mathrm{v}(3.1)$ \\
\hline Chemicals & 30 (14.5) & $26(13.7)$ & $3(4.3)$ & $1(2.6)$ & $0(0.0)$ & $2(1.3)$ & $6(2.0)$ & $15(2.3)$ \\
\hline Total & $207(100.0)$ & $190(100.0)$ & $69(100.0)$ & $60(100.0)$ & $97(100.0)$ & $150(100.0)$ & $295(100.0)$ & $649(100.0)$ \\
\hline
\end{tabular}

*Column percentage

†Significant association between poison types and sexes for each age group $(p<0.0001)$.

( $n=1213,71.6 \%$ ). In terms of morbidity and mortality, the majority stayed in hospitals for fewer than four days ( $\mathrm{n}=1580,93.2 \%)$, and there were 98 deaths $(5.8 \%)$ caused by poisoning from the four major types of poisons in NSW in 2000.

To further elucidate the relationship between different poison types and age and sex, poison types were cross tabulated by age groups and sex. Table 3 presents the results of poison types by age groups and sex. There was a significant association between poison types and age groups $\left(\chi_{9}^{2}=517.6, p<0.001\right)$ with analgesics more related to the 0-4 and 15-19 years groups. Psychotropic drugs tended to be associated with those aged 15-19 years, venoms with those 5-9 years, and chemicals with those 0-4 years. The association between poison types and sex varied across different age groups. There was no significant association between types of poison and sex for children younger than 10 years. On the other hand, significant associations between poison types and sex were found for both those aged 10-14 years $\left(\chi_{3}^{2}=59.4, p<0.001\right)$ and $15-19$ years $\left(\chi_{3}^{2}=44.3\right.$, $\mathrm{p}<0.001$ ). Significantly higher proportions of females in both age groups tended to be admitted to hospital because of analgesics poisoning than males, whereas more males tended to be admitted because of venom poisoning (table 3 ).

The results on the standardised incidence ratios for metropolitan and rural hospitalisations and different poison types stratified by age and sex were tabulated (table 4). The standardised incidence ratio of poisoning related hospitalisation between metropolitan and rural areas compared with the NSW average, varied across different poison types. There were few differences between metropolitan and rural areas for analgesic and chemical related poisoning admissions across different age groups and sex. However, differences in standardised incidence ratios between geographic locations for psychotropic and venom related poisoning admissions were noted (table 4 ). The standardised hospitalisation rates for rural regions were significantly higher than the NSW average for both poison types across most age groups and sex.

In terms of the outcome of hospitalisation, there was an association between poison type and length of stay in hospital with a higher proportion of those poisoned by analgesics staying more than three days compared with other poison types $\left(\chi^{2}{ }_{3}=28.1, \mathrm{p}<0.001\right)$. Among those who were poisoned by analgesics, all children less than 10 years stayed less than four days in the hospital, whereas $12.4 \%$ of $15-19$ years stayed more than three days. In terms of mortality, comparisons of the standardised mortality ratios for both metropolitan and rural areas across different age groups and sex indicated that there was no significant difference for most age and sex subgroups, except for females of the 10-14 year old group (table 4).

\section{DISCUSSION}

This study aimed to test the hypothesis that there is an association between poison types and geographic locations across different age groups and sex. The results obtained in this study do not provide strong evidence to refute the hypothesis. In fact, different conclusions can be drawn from the results according to different poison types.

Of the four different poison types examined in this study, there were no significant differences in the standardised incidence ratios of hospitalisation between metropolitan and rural areas, when compared to the NSW average, for two types, analgesics and chemicals. These two are the major types of medicinal and non-medicinal substances that are associated with most incidences of poisoning. ${ }^{10}{ }^{14}$ On the other hand, significant differences in standardised incidence ratios of hospitalisation between metropolitan and rural areas across nearly all age groups and sex were observed for psychotropic drugs and venom poisoning. For these two particular types of substance, the results provide some evidence to support the hypothesis.

In referring to the basic assumption of the hypothesis that the association between poison type and geographic location is based on accessibility and exposure, one would expect hospitalisations caused by chemical related and venom related poisoning to be more prevalent in rural areas. The results obtained on venom seem to provide support to the hypothesis that children and adolescents in rural areas are more exposed to insects, spiders, and snakes and hence have a higher chance of being bitten and require hospitalisation. However, the results obtained on chemical related poisoning do not support the hypothesis. In interpreting this particular result, one may speculate that children and adolescents are exposed to chemicals similarly in both metropolitan and rural areas, thus the chances of poisoning are also similar. This requires further investigation into the degree of exposure in both areas. The same argument can also apply to analgesics.

The results obtained on psychotropic medication, that would require a prescription from a medical practitioner according to the law in NSW, are unexpected and interesting. The standardised incidence ratios of hospitalisations due to psychotropic drug poisoning are consistently larger in rural areas and significantly different from the state average across nearly all age groups and sex. Should the assumption of exposure for the hypothesis under testing be true, the results on psychotropics suggest that there are greater exposures to this type of medicinal substances especially among children in the rural areas of NSW than their metropolitan counterparts. This may probably be due to many different factors, as suggested by some researchers, that include greater availability, different parental attitudes towards poisonous substances, poorer home design, and a lack of information resources and education..$^{10}$ Due to the lack of information on the prescriptions of psychotropic medications in NSW for both metropolitan and rural areas, the assertion of such an interpretation is difficult. The result obtained for $15-19$ year olds showing that the standardised incidence ratios of hospitalisation is significantly larger than the state average only for rural females, seems to suggest the possibility of intentional self harm via psychotropic medications. There is some supportive evidence showing that psychotropic medica- 


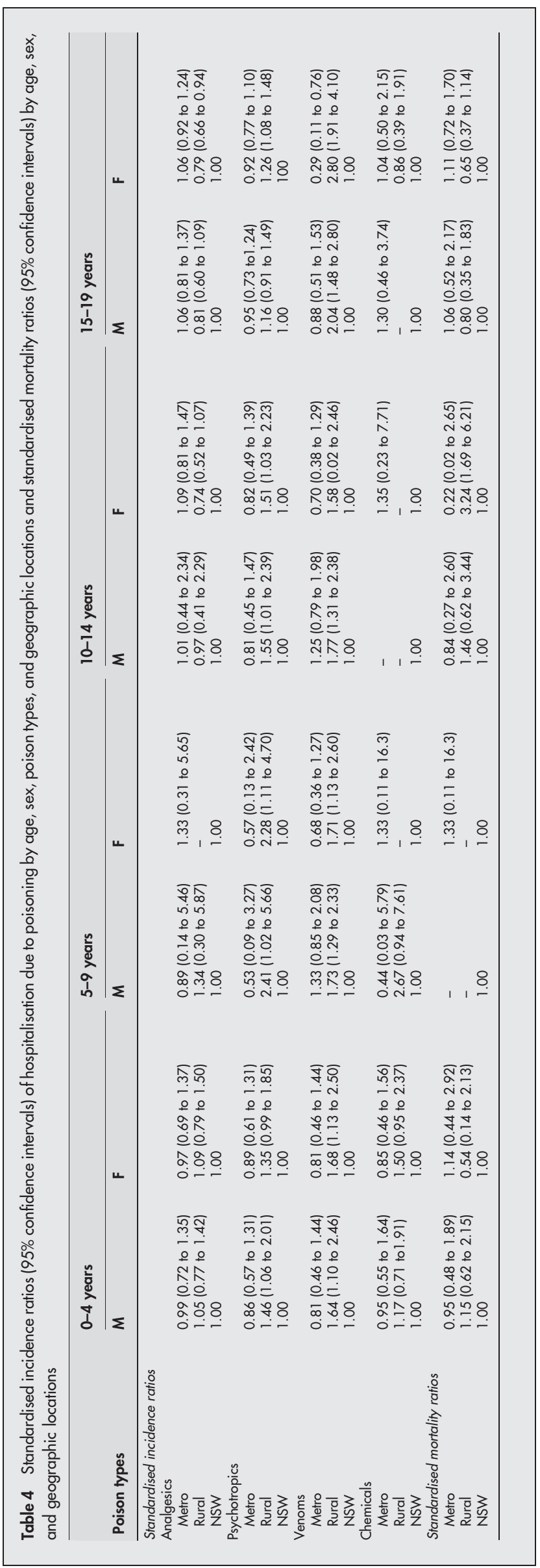

tions are the most common substances of choice for suicide and self harm among females in Australia. ${ }^{22}$ It could also be possible that the higher rate of hospitalisation is associated with accidental overdose of antidepressive medications. It has also been suggested that the prevalence of depression is much higher in rural areas, especially among females, than in urban areas. ${ }^{23}$ However, because of the lack of information on the intention of these poisoning incidents, it is difficult to determine the reason for the differences in hospitalisation rates.

The results obtained on the association between poison types and age are consistent with previous studies mentioned in the introduction. It has been recognised that the age difference in different types of poisoning is mainly due to the differences in developmental characteristics between children and adolescents. ${ }^{14} 15$ For example, poisoning in children under the age of 5 is most likely to be accidental but most likely to be deliberate in older adolescents. This is partially reflected in the results found on the length of stay and age group among those poisoned with analgesics. The lengthier stay in hospitals for adolescents, reflecting a more serious condition, may probably be due to intentional self harm. The results of this study further suggest that accessibility and exposure to different poisons may interact with age in determining the occurrence of poisoning incidents. This requires further investigation.

There are practical implications of the results obtained from this study. Given the love of outdoor activities in Australia, especially in rural areas, and the well known danger of some Australian snakes and spiders, the finding of this study is of particular importance. Due to the higher chances of exposure to wild animals, snakes and insects in the rural areas, greater attention should be paid in providing education and information to rural children and adolescents in the identification as well as proper handling of these creatures. Appropriate safety devices should be installed to protect against venomous insects, spiders, and snakes in homes and schools. Further investigations are urgently needed to examine risk factors for the increased risk of hospitalisations caused by psychotropic medication poisoning among rural children. The issue of availability of these medications in rural areas may be associated with prescription practices and also requires urgent examination.

Several limitations have been identified in this study. First, data utilised in this study are confined to hospitalisation only. Due to the lack of coordinated data collection mechanism for emergency presentations in NSW, data on emergency presentation of poisoning incidents are not available. The results obtained represent the more severe cases of poisoning. Second, there may be potential bias due to misclassification of poison cases. This is a very common source of bias in studies that utilise population based vital statistical data such as hospitalisation and death. The quality of data used in this study can, to a certain degree, be assured because they have been collected via a standardised mechanism and have used well trained medical information personnel. Nevertheless, misdiagnosis may still occur in cases of multiple drugs poisoning or poisoning due to substances not easily identified at the time of admission. Underreporting may occur if these cases are misdiagnosed, and biased estimation of risk will result. In relation to this is the misclassification of the geographic location since it is classified by the residential postcode of the patient. It could be possible that urban children and adolescents were admitted to rural hospitals, especially for venom related poisoning. These patients could have resided in urban areas, but were bitten by snakes, spiders, or other insects in rural areas and hence were admitted to rural hospitals. However, due to the lack of information on these cases, it is difficult to 


\section{Key points}

- There is some evidence for an association between poison type and geographic location for psychotropic and venom related poisoning.

- There is also a need to examine risk factors for increased risk of poisoning caused by psychotropic medications among rural children.

- There is an urgent need to provide education and information to rural children and adolescents in the identification and proper handling of insects, spiders, and snakes.

estimate the impact of this potential bias on the overall risk calculation. Third, there is potential bias in the possibility of differential admission rates between urban and rural hospitals. It would be possible that urban hospitals, due to their larger capacities and having designated children's facilities, are more likely to admit young patients than rural hospitals. Should this be true, the equal hospitalisation rates of poisoning among children would become significant. Fourth, there is also potential bias in the calculation of standardised incidence ratios and standardised mortality ratios. The calculations of these two parameters and their corresponding 95\% confidence interval in this study are based on the assumption of an underlying unbiased Poisson distribution probability model. ${ }^{21}$ As discussed above, the lack of information on the levels of exposure to different poison types may have constituted a bias to the study. The results obtained in this study may have been over or under-estimated.

\section{REFERENCES}

1 United Nations Children' Fund. A league table of children deaths by injury in rich nations. Innocenti report card, issue No 2. Florence: Innocenti Research Centre, 2001

2 Cox E, Yseng DS, Powell I. Trends in falls, poisoning, drowning, and burns in Wisconsin: 1986-1996. Wis Med J 2001;100:39-42.
3 Woolf A, Alpert HR, Garg A, et al. Adolescent occupational toxic exposures: a national study. Arch Pediatr Adolesc Med 2001;155:704-10.

4 Litovitz TL, Klein-Schwartz W, Rodgers GC Jr, et al. Annual report of the American Association of Poison Control Centres Toxic Exposure Surveillance System. Am J Emerg Med 2002;20:391-452.

5 Davis LE, Benson BE, Easom LA, et al. Methanol poisoning exposures in the United States: 1993-1998. J Toxicol Clin Toxicol 2002;40:499-505.

6 Thompson JP, Casey PB, Vale JA. Suspected paediatric pesticide poisoning in the UK. II: Home Accident Surveillance System 1989-1991, Hum Exp Toxicol 1994; 13:534-6.

7 Pearn J, Nixon, Ansford A, et al. Accidental poisoning in childhood: five year urban population study with 15 year analysis of fatality. BMJ 1984;288:44-6.

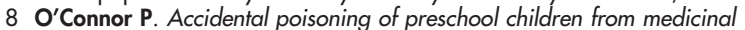
substances. Injury research and statistics series number 9. Canberra, Australia: Australian Institute of Health and Welfare, 2001.

9 Hoy JL, Day LM, Tibballs J, et al. Unintentional poisoning hospitalisations among young children in Victoria. Inj Prev 1999;5:31-5.

10 Reith DM, Pitt WR, Hockey R. Children poisoning in Queensland: an analysis of presentation and admission rates. J Paediatr Child Health 2001;37:446-50

11 London L, Bailie R. Challenges for improving surveillance for pesticide poisoning: policy implications for developing countries. Int J Epidemiol 2001;30:564-70.

12 Ahmed N, Andersson R. Differences in cause-specific patterns of unintentional injury mortality among 15-44-year-olds in income-based country groups. Acidc Anal Prev 2002;34:541-51.

13 Ab Rahman AF. Drug and chemical poisoning admissions at a teaching hospital in Malaysia. Hum Exp Toxicol 2002;21:377-81.

14 Riordan M, Rylance G, Berry K. Poisoning in children 2: painkillers. Arch Dis Child 2002;87:397-9.

15 Riordan M, Rylance G, Berry K. Poisoning in children 3: common medicine. Arch Dis Child 2002;87:400-2.

16 McGuigan MA. Common culprits in childhood poisoning: epidemiology, treatment and parental advice for prevention. Paediatric Drugs 1999;1:313-24.

17 James LP, Abel K, Wilkinson J, et al. Phenothiazine, butyrphenone, and other psychotropic medication poisonings in children and adolescents. J Toxicol Clin Toxicol 2000;38:615-23.

18 NSW Department of Health. Inpatient statistics collection. Available at http:// internal.health.nsw.gov.au/im/ims/isc/ (accessed June 2003).

19 HCIA. ICD-9-CM. International classification of diseases. 9th Revision clinical modification. Maryland: HCIA Inc, 1993.

20 Australian Bureau of Statistics. Population by age and sex, NSW. ABS catalogue No 3235.1. Canberra, Australia: Australian Commonwealth Government, 2000.

21 Rothman KJ, Greenland S. Modern epidemiology. 2nd Ed. Philadelphia: Lippincott Williams \& Wilkins, 1998.

22 Steenkamp M, Harrison J. Suicide and hospitalised self-harm in Australia. Injury Research and Statistics Series. Canberra, Australia: Australian Institute of Health and Welfare, 2000

23 Patton G, Burns J. Preventive interventions for youth suicide: a risk factor approach. Report prepared for the National Health and Medical Research Council. Canberra, Australia: Centre for Adolescent Health, 1998. 